\title{
Linx
}

Revue des linguistes de l'université Paris X Nanterre

39 | 1998

Modèles linguistiques : convergences, divergences

\section{DI/PL, Linéarisation, Arbres Polychromes : trois approches de l'ordre des mots}

ID/LP, Linearization, Polychrome Trees: three approaches to word order

\section{Olivier Bonami}

\section{(2) OpenEdition}

\section{Journals}

Édition électronique

URL : http://journals.openedition.org/linx/873

DOI : $10.4000 / \operatorname{lin} x .873$

ISSN : 2118-9692

\section{Éditeur}

Presses universitaires de Paris Nanterre

\section{Édition imprimée}

Date de publication : 15 décembre 1998

Pagination : 43-70

ISSN : 0246-8743

\section{Référence électronique}

Olivier Bonami, «DI/PL, Linéarisation, Arbres Polychromes : trois approches de l'ordre des mots », Linx [En ligne], 39 | 1998, mis en ligne le 27 juin 2012, consulté le 20 avril 2019. URL : http:// journals.openedition.org/linx/873 ; DOI : 10.4000/linx.873

Ce document a été généré automatiquement le 20 avril 2019.

Département de Sciences du langage, Université Paris Ouest 


\title{
DI/PL, Linéarisation, Arbres Polychromes : trois approches de l'ordre des mots*
}

\author{
ID/LP, Linearization, Polychrome Trees: three approaches to word order
}

\section{Olivier Bonami}

1 Une des tâches centrales de toute théorie syntaxique est de spécifier la relation entre la

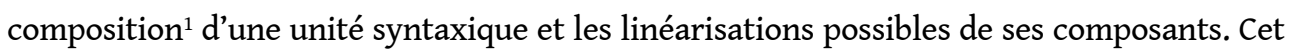
article présente une comparaison de trois approches distinctes de cette relation dans le cadre général des grammaires syntagmatiques : les grammaires DI/PL (Gazdar et al. 1985), les grammaires d'arbres polychromes (GAP, Cori \& Marandin 1993), et les grammaires de linéarisation directe (GLD, Kathol 1995). La comparaison sera basée sur l'examen de deux domaines empiriques réputés récalcitrants, et qui mettent au jour les différences entre les approches: le placement des adverbes dans un GV en français (section 2), et l'ordre dans les phrases finies en allemand (section 3). Dans les deux cas, les données observées amènent à analyser un certain type de syntagme comme une séquence strictement ordonnée de domaines d'ordre ; cette conception de l'ordre est difficile à réconcilier avec une analyse DI/PL, alors qu'elle est encodée directement dans les grammaires d'arbres polychromes et dans les grammaires de linéarisation directe. La comparaison entre GAP et GLD est plus délicate, mais une piste est explorée en conclusion.

2 Il est difficile de comparer plusieurs approches de l'ordre sans entrer dans des questions ayant trait aux cadres grammaticaux dans lesquels ces approches sont enchâssées. Afin de contourner cette difficulté, j'ai choisi d'utiliser HPSG (Pollard \& Sag 1987, 1994) comme unique architecture grammaticale, et d'étudier les trois approches de l'ordre comme trois alternatives possibles dans ce cadre général. Ce choix implique de disposer d'une traduction des GAP dans le formalisme de structures de traits typées de HPSG; une reconstruction est proposée en annexe. 


\section{Présentation des trois approches}

\subsection{Les grammaires $\mathrm{DI} / \mathrm{PL}$}

Les grammaires syntagmatiques classiques font une hypothèse maximale sur la relation entre composition et linéarisation: la composition d'une expression détermine complètement sa linéarisation. Dans une grammaire syntagmatique classique, il est donc impossible de dire que deux unités qui ne présentent pas le même ordre des mots ont la même structure de composition. Par exemple, (1a) et (1b) ne sont pas analysées à l'aide des mêmes règles : le GV de (1a) est obtenu en utilisant (2a), alors que celui de (1b) est obtenu en utilisant (2b).

\begin{tabular}{|l|l|l|l|}
\hline (1) & a. & Paul offrira ce livre à Marie & b. Paul offrira à Marie ce livre \\
\hline
\end{tabular}

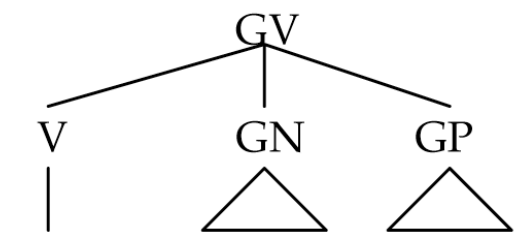

offrira ce livre à Marie

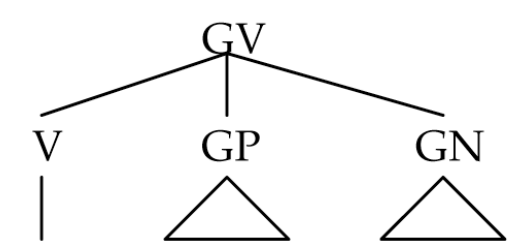

offrira à Marie ce livre

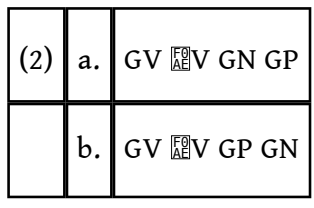

Les grammaires DI/PL (Gazdar et al.1985) modifient ce tableau en découplant complètement la structure en constituants de l'ordre des mots. Elles distinguent les règles de dominance immédiate (DI) des règles de précédence linéaire (PL), ce qui permet qu'une seule structure de composition soit compatible avec différents ordres linéaires des constituants. Une règle de dominance spécifie la composition d'un certain type de syntagme sans imposer de relation d'ordre entre les composants. Par exemple, les deux ordres dans le GV de (1) sont obtenus à partir de l'unique règle (3a). Une règle d'ordre de la forme $\mathrm{X}<\mathrm{Y}$ spécifie que, si deux unités de type $\mathrm{X}$ et $\mathrm{Y}$ sont des constituants immédiats d'un certain syntagme, l'unité de type $\mathrm{X}$ doit précéder l'unité de type $Y$. Par exemple, les règles (3b) spécifient que certains ordres sont interdits dans le GV : le verbe doit être en tête.

\begin{tabular}{|c|c|c|}
\hline (3) & a. & $\mathrm{GV}$ 㜀 $\mathrm{V}, \mathrm{GN}, \mathrm{GP}$ \\
\hline & b. & $\begin{array}{l}\mathrm{V}<\mathrm{GN} \\
\mathrm{V}<\mathrm{GP}\end{array}$ \\
\hline
\end{tabular}


Les grammaires DI/PL autorisent donc une relation non-fonctionnelle entre composition et linéarisation, tout en imposant de fortes contraintes sur cette relation. Ces contraintes peuvent être résumées sous la forme suivante :

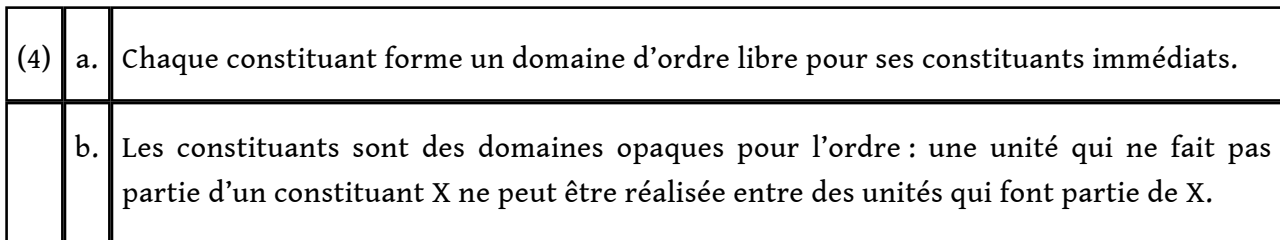

6 Par domaine d'ordre libre, j'entends un domaine dans lequel l'ordre entre les constituants est potentiellement libre, et peut être contraint par des règles d'ordre. Il se peut donc que l'ordre à l'intérieur d'un constituant soit complètement fixe ; par exemple, en français, l'ordre dans un GV constitué de V et GN est fixe. Le point important est qu'en l'absence de règles d'ordre spécifiques, l'ordre à l'intérieur d'un constituant est libre, alors que l'ordre entre des constituants qui ne sont pas dominés par la même mère est contraint. Pour illustrer ces propriétés, prenons une grammaire abstraite qui ne contient aucune règle d'ordre :

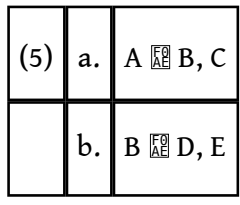

7 Cette grammaire autorise les séquences (6a), mais pas les séquences (6b):

$$
\begin{array}{|l|l|l|}
\hline \text { (6) } & \text { a. } & \text { CDE, CED, DEC, EDC } \\
\hline & \text { b. } & \text { * DCE, }{ }^{*} \mathrm{ECD} \\
\hline
\end{array}
$$

8 D et $\mathrm{E}$ formant un constituant, ils appartiennent à un même domaine d'ordre libre ; de même, $\mathrm{B}$ et $\mathrm{C}$ forment un domaine d'ordre libre. Mais, crucialement, $\mathrm{D}$ et $\mathrm{E}$ ne sont pas des éléments de ce domaine d'ordre libre, ce qui explique pourquoi $\mathrm{C}$ ne peut pas être réalisé entre $\mathrm{D}$ et $\mathrm{E}$.

\subsection{Les grammaires d'arbres polychromes}

9 Les grammaires d'arbres polychromes (Cori \& Marandin 1993) constituent un formalisme d'analyse syntaxique fondé sur une structure syntagmatique particulière. Dans ce cadre, on considère qu'un syntagme est constitué d'une série de positions, c'est-à-dire de séquences de constituants strictement ordonnées entre elles. En ce sens, la relation entre composition et linéarisation dans une GAP n'est pas la même que dans une grammaire DI/ PL. (4a) est remplacé par (7) :

(7) Un constituant est une séquence strictement ordonnée de domaines d'ordre libre. 

sémantiques ou pragmatiques, mais on ne préjuge pas des propriétés pertinentes; en particulier, rien n'impose que l'occupation d'une position soit liée à une fonction grammaticale. obligatoirement instanciée dans tout type de syntagme, et n'est instanciée que par un seul constituant ${ }^{2}$. Par exemple, on peut proposer les analyses suivantes pour les groupes verbaux de (1) :

$12(8)$

$a$.

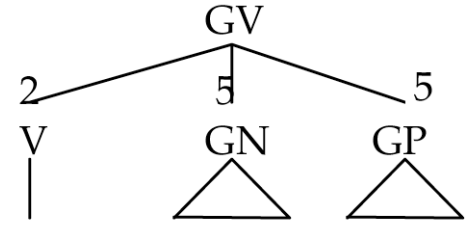

offrira ce livre à Marie $b$

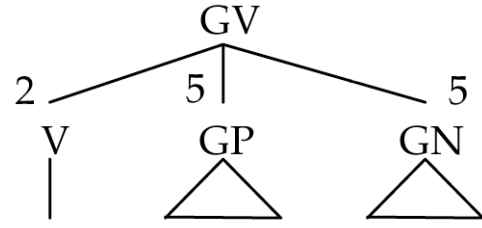

offrira à Marie ce livre

13 Les différentes positions sont représentées par les couleurs attachées au branches de l'arbre; deux branches ayant la même couleur dominent deux constituants occupant la même position. Par convention, on note les couleurs par des entiers naturels, et on suppose que l'ordre des couleurs correspond à l'ordre entre les positions.

Dans les arbres (8), la position pivot est la position 2, qui est, dans un GV, obligatoirement instanciée par le verbe conjugué tête du syntagme. Les positions 3 et 4 ne sont pas occupées. La position 5 est occupée par deux constituants, le GN un livre et le GP à Marie.

Une grammaire d'arbres polychromes est constituée d'une collection d'arbres élémentaires (arbres de profondeur 1) à partir desquels on peut obtenir des arbres complexes en utilisant une opération de composition. L'opération de composition est sensible à l'identité des pivots; spécifiquement, si un syntagme a pour pivot un constituant de même catégorie que lui, et si ce pivot est lui-même un constituant syntagmatique, alors la composition a un effet de compactage: l'arbre composé est " aplati ", et les filles de la mère et celles de son pivot se retrouvent au même niveau. on essaye de composer (9a) avec le pivot de $(9 b)$, on obtient l'arbre $(9 c=8 a)^{3}$ :

$17 \quad(9)$

a.<smiles></smiles>

$b$.

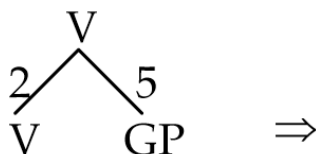

C.

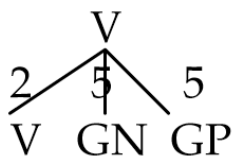

L'opération de composition avec compactage permet donc d'obtenir une structure syntagmatique plate pour les groupes verbaux en n'utilisant que des arbres élémentaires à branchement binaire.

Un effet intéressant de la composition avec compactage est illustré en (11) : dans cet exemple, on tente de composer un arbre dominant une branche 5 dans le pivot d'un arbre dominant une branche 4 . 
<smiles>[B]C=CC</smiles><smiles>CC</smiles><smiles>[2H]C=CC</smiles><smiles>[2H]C=C</smiles>

Dans l'arbre résultant, la branche 5 se retrouve à droite de la branche 4, en accord avec l'ordre intrinsèque des positions. Le compactage a donc pour effet, dans certains cas, un réordonnancement des branches.

L'exemple (11) illustre le fait que dans une analyse GAP, les arbres élémentaires intervenant dans la composition d'un arbre complexe ne correspondent pas nécessairement à des constituants. Les arbres élémentaires jouent le rôle de descriptions partielles des constituants, la description complète n'étant obtenue qu'après compactage. Cette perspective sur les arbres élémentaires implique que, malgré l'existence d'effets de réordonnancement des branches, les analyses GAP partagent avec les analyses DI/PL la caractéristique $(4 \mathrm{~b})$ : les constituants sont des domaines opaques pour l'ordre.

Je présente en annexe une reconstruction des GAP dans le formalisme des structures de traits typées, qui permet d'utiliser la théorie de l'ordre enchâssée dans les GAP en conjonction avec l'ensemble des outils analytiques développés en HPSG.

\subsection{Les grammaires de linéarisation directe}

24 Afin de rendre compte de l'ordre des mots dans la phrase en allemand, Kathol \& Pollard (1995) et Kathol (1995) proposent une modification majeure de l'architecture de HPSG, qui altère considérablement la notion de structure en constituants ${ }^{4}$.

La grammaire de Kathol peut être caractérisée comme une grammaire de linéarisation directe (GLD). La composition d'un syntagme fait l'objet de deux représentations indépendantes : à côté de sa structure en constituants, éventuellement hiérarchisée, un constituant est associé à un domaine d'ordre qui encode une représentation linéaire des unités entrant dans sa composition. Alors que les éléments de la structure en constituants ne sont pas ordonnés entre eux, des règles de précédence linéaire ordonnent les membres du domaine d'ordre.

Une analyse possible du GV de (1a) dans une GLD est donnée en (12) : 


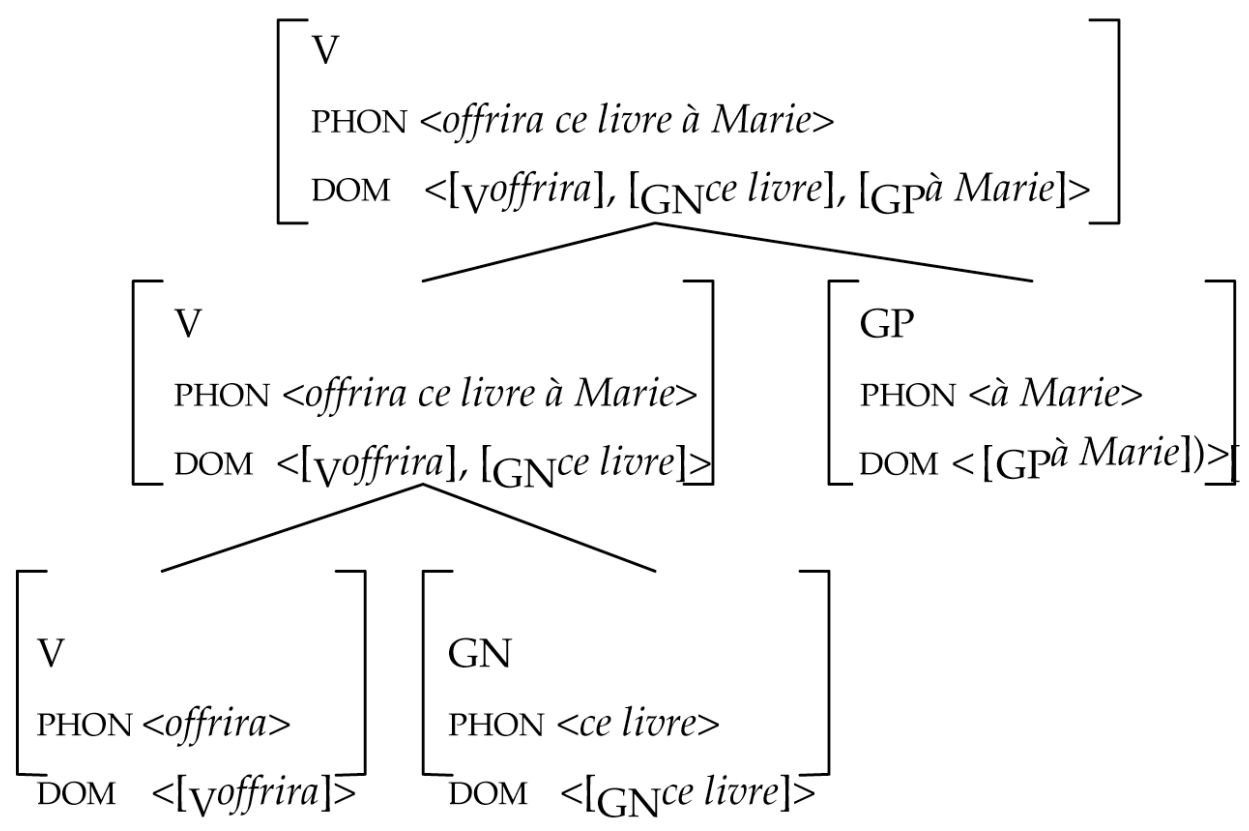

Le verbe offrira est d'abord composé avec le GN un livre, pour donner un signe de catégorie GV ayant ces deux signes dans son domaine d'ordre. Ce nouveau signe est ensuite composé avec le GP à Marie, pour donner un nouveau signe de catégorie GV, dont le domaine d'ordre est un mélange de ceux de ses deux composants. Le résultat est que les trois unités offrira, un livre et à Marie sont présents dans le domaine d'ordre du GV, et susceptibles, à priori, d'apparaître dans n'importe quel ordre.

Les domaines d'ordre au sens des GLD ne sont cependant pas des domaines d'ordre libre. Chaque domaine est structuré en une séquence de champs topologiques. Les champs topologiques dans une GLD jouent un rôle voisin de celui des positions dans une GAP : ce sont des domaines d'ordre libre strictement ordonnés entre eux.

Formellement, la notion de champ topologique est encodée directement dans la définition des objets de domaines, c'est-à-dire des objets qui apparaissent dans les domaines d'ordre. Kathol suppose que les objets de domaines regroupent trois informations distinctes : ils spécifient non seulement la forme phonologique (PHON) et la catégorie (SYNSEM) de l'unité, mais également une information sur le champ topologique dans lequel l'unité va apparaître (TOPO).

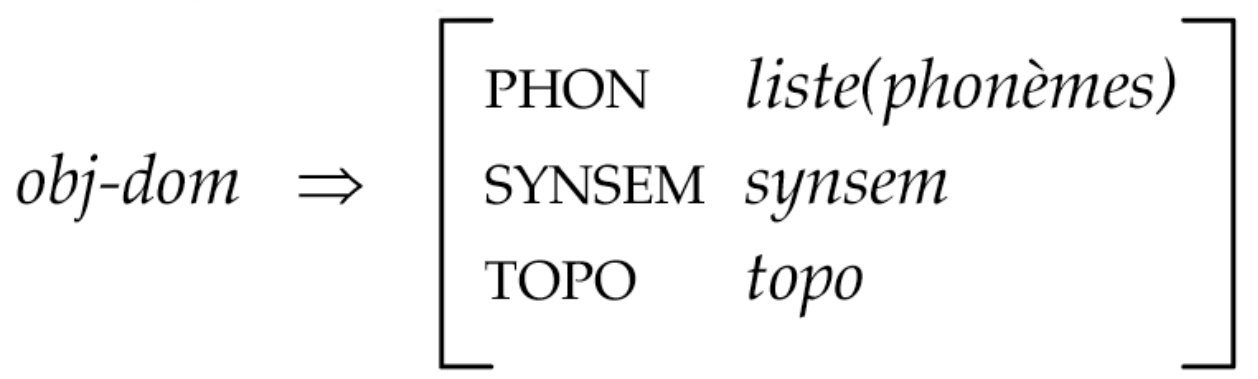

32 Le choix des valeurs possibles pour TOPO est susceptible de varier en fonction du type de syntagme analysé. Pour les GV de (1), on peut supposer que TOPO peut prendre au moins 
deux valeurs distinctes, initial et final. L'ordre strict entre les champs topologiques est obtenu en ordonnant les objets de domaine en fonction de leur valeur pour TOPO :

(14) $[$ TOPO initial $]<$ [TOPO final $]$

d'ordre référant aux catégories syntaxiques. Les contraintes
obtenues directement en utilisant des contraintes sur l'assignation
Spécifiquement, les quatre contraintes suivantes sont suffisantes
\begin{tabular}{|l|l|l|}
\hline$(15)$ & a. & Un objet de catégorie GN est TOPO final. \\
\hline & b. & Un objet de catégorie GP est TOPO final. \\
\hline & c. & Un objet de catégorie V est TOPO initial. \\
\hline & d. & Dans une phrase, il y a exactement un objet [TOPO initial]. \\
\hline
\end{tabular}




\section{Premier exemple : l'ordre dans le GV en français}

\subsection{Une analyse DI/PL de l'ordre dans le GV}

Dans deux articles récents, Anne Abeillé \& Danièle Godard (1998, à paraître) proposent une analyse détaillée de l'ordre des constituants dans le groupe verbal en français, formulée dans le cadre d'une grammaire DI/PL. Je rappelle ici les éléments essentiels de leur analyse, avant d'introduire quelques données supplémentaires problématiques.

Abeillé \& Godard supposent que la structure en constituants des GV à verbe fini est plate ; en particulier, si le GV contient un auxiliaire, l'auxiliaire et le ou les participes apparaissent au même niveau, et au même niveau que les compléments. De même, l'ensemble des ajouts apparaissant à l'intérieur d'un GV à verbe fini sont des constituants immédiats de ce GV. L'analyse du GV de (17) est donc comme en (18) :

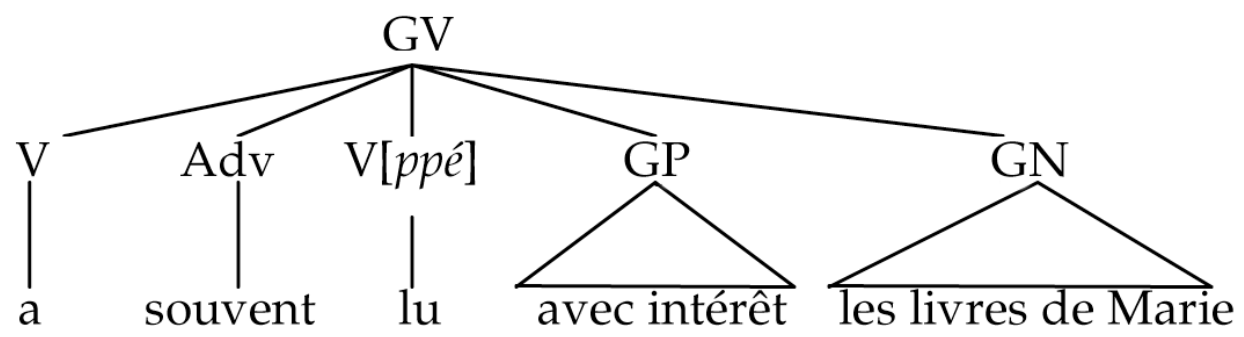

(19)

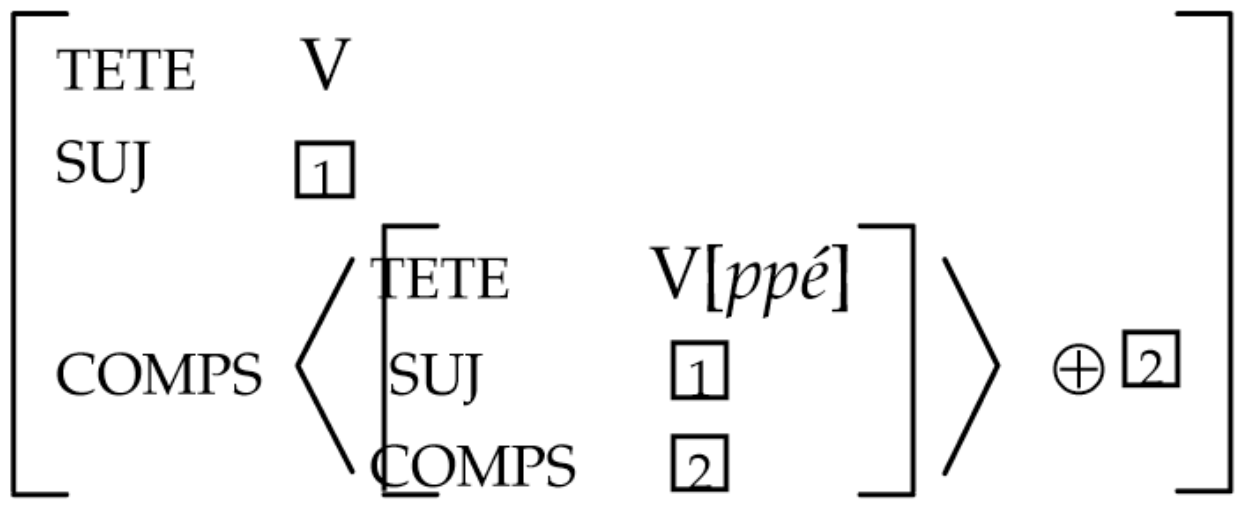

$41 \quad(20)$

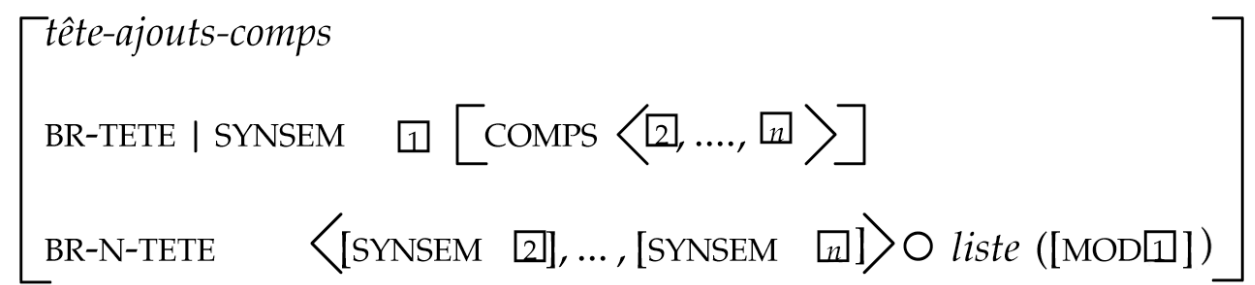

(18) est une instance de ce schéma: le verbe tête a prend le participe lu comme complément, et hérite de son complément d'objet les livres de Marie. Deux ajouts souvent et avec intérêt sont librement ajoutés dans le GV. J'admets les justifications proposées par 
Abeillé \& Godard (1996, à paraître) pour cette analyse, pour me concentrer sur le traitement de l'ordre.

43 Je considère d'abord les GV sans ajouts, pour aborder ensuite les problèmes spécifiques liés aux adverbes. Dans un GV sans adverbes, Abeillé \& Godard observent que les règles d'ordre sont sensibles au "poids" des constituants. Au premier abord, tous les constituants lexicaux doivent précéder tous les constituants syntagmatiques : le verbe conjugué et les éventuels participes sont à gauche des compléments syntagmatiques (21-22). Les compléments nominaux des verbes supports doivent apparaître à gauche des compléments syntagmatiques s'ils sont lexicaux (23). Par contre, ils peuvent apparaitre à leur droite s'ils sont syntagmatiques (24):

\begin{tabular}{|l|l|l|}
\hline$(21)$ & Jean lit un livre $/ *$ Jean un livre lit \\
\hline$(22)$ & Jean a lu un livre $/$ * Jean a un livre lu \\
\hline$(23)$ & a. & Le président a rendu hommage à son prédécesseur \\
\hline & b. & * Le président a rendu à son prédécesseur hommage \\
\hline$(24)$ & a. & Le président a rendu un hommage appuyé à son prédécesseur \\
\hline & b. & Le président a rendu à son prédécesseur un hommage appuyé \\
\hline
\end{tabular}

44 Cependant, le paramètre qui est en jeu dans ces exemples ne peut pas être, à strictement parler, le statut lexical ou syntagmatique des constituants; ainsi, on a vu que le complément lexical hommage en (23) doit être ordonné avant les compléments syntagmatiques. Mais il n'en va pas de même pour un complément d'objet constitué d'un nom propre, qui est pourtant lui aussi lexical :

\begin{tabular}{|l|l|l|}
\hline$(25)$ & a. & Jean a présenté Alexandre à sa sœur \\
\hline \hline & b. & Jean a présenté à sa sœur Alexandre \\
\hline
\end{tabular}

Il faut donc admettre que certains constituants, bien que lexicaux, sont considérés comme des constituants syntagmatiques du point de vue de l'ordre des mots. Abeillé \& Godard rendent compte de ce fait en introduisant un trait [LEGER \pm$]^{8}$; les mots peuvent être lexicalement légers, comme les noms communs, ou non-légers, comme les noms propres. Les syntagmes sont (presque) toujours non-légers.

46 Abeillé \& Godard proposent donc une règle qui ordonne les constituants légers à gauche des constituants non-légers. Une règle supplémentaire est nécessaire pour rendre compte $\mathrm{du}$ fait que, alors que l'ordre entre les constituants syntagmatiques est libre, l'ordre entre les constituants lexicaux est contraint.

$$
\begin{array}{|l|l|l|}
\hline(26) & \text { a. } & {[\text { LEGER }+]<[\text { LEGER - }]} \\
\hline
\end{array}
$$


$[$ COMPS $<\ldots,[1], \ldots>]<[1]$

47 La règle (26b) rend donc compte du fait que l'auxiliaire précède le participe, et que le complément nominal des verbes support précède le verbe support :

(27) Jean a parlé / * Jean parlé a

(28) Jean m'a rendu hommage / * Jean m'a hommage rendu

Passons maintenant aux adverbes. Ceux-ci sont répartis dans deux classes : les adverbes non-légers et les adverbes légers. Quelques exemples de chaque classe sont donnés en (29).

\begin{tabular}{|l|l|l|l|}
\hline (29) & a. & \multicolumn{2}{|l|}{$\begin{array}{l}\text { Adverbes non-légers } \\
\text { probablement, souvent, volontairement, attentivement, etc. }\end{array}$} \\
\hline \hline & b. & Adverbes légers & \\
\hline & & (i) & bien, mal, mieux, beaucoup, etc. \\
\hline & & (ii) & rien, tout, (les-) tous, etc. \\
\hline
\end{tabular}

Les quantifieurs nus sont traités comme des mots portant, comme les adverbes au sens strict, la spécification $[\mathrm{ADV}+]$, ce qui permet de rendre compte du fait que les deux classes d'unités ont la même distribution.

Dans le GV fini, les adverbes légers doivent apparaître avant les compléments non-légers :

\begin{tabular}{|l|l|l|}
\hline$(30)$ & a. & Jean a bien parlé à Marie $/{ }^{*}$ Jean a parlé à Marie bien \\
\hline & b. & Jean a tout dit à Marie $/{ }^{*}$ Jean a dit à Marie tout \\
\hline \hline$(31)$ & a. & Jean a parlé à Marie admirablement bien \\
\hline & b. & Jean a dit à Marie absolument tout \\
\hline
\end{tabular}

51 La règle (26a) suffit pour rendre compte de cette observation ${ }^{9}$. Les choses se compliquent quand on prend en compte les adverbes non-légers. Ceux-ci peuvent apparaître après les constituants légers, mais également entre le verbe tête et les autres constituants légers. Par exemple, en (32b), gentiment est linéarisé entre le verbe conjugué et le participe :

(32) a. $*$ Jean gentiment a lu ce livre ${ }^{10}$ 


\begin{tabular}{|l|l|l|}
\hline & b. & Jean a gentiment lu ce livre à sa grand-mère \\
\hline \hline & c. & Jean a lu gentiment ce livre à sa grand mère \\
\hline & d. & Jean a lu ce livre gentiment à sa grand-mère \\
\hline \hline & e. & Jean a lu ce livre à sa grand-mère gentiment \\
\hline
\end{tabular}

Pour rendre compte de la distribution des adverbes non-légers, Abeillé \& Godard révisent les règles (26) de manière à ce que seuls les constituants non-légers non-adverbiaux précèdent les constituants légers :

\begin{tabular}{|l|l|l|}
\hline (33) & a. & BRANCHE-TETE < BRANCHE-N-TETE \\
\hline \hline & b. & {$[$ LEGER + $]<[$ LEGER - , ADV - $]$} \\
\hline & c. & {$[$ COMPS <..., [1],... $]<[1][$ ADV - $]$} \\
\hline
\end{tabular}

53 (33a) rend compte du fait que le verbe tête est toujours premier ; (33b) assure que seuls les constituants non-adverbiaux sont soumis à la règle qui ordonne les légers avant les non-légers. Enfin, (33c) est inchangée ${ }^{11}$.

54 L'analyse d'Abeillé \& Godard règle une bonne partie des problèmes liés à l'ordre des constituants dans le groupe verbal; mais il est un problème qui est laissé ouvert: les règles proposées par Abeillé \& Godard laissent le placement des adverbes non-légers par rapport aux constituants légers complètement libre. Il existe cependant des contraintes ; par exemple, un adverbe non-léger ne peut pas être réalisé entre l'adverbe léger mieux et le participe passé :

\begin{tabular}{|l|l|l|}
\hline$(34)$ & a. & * Paul a mieux fréquemment lu ce texte \\
\hline & b. & Paul a mieux lu ce texte fréquemment \\
\hline & c. & Paul a fréquemment mieux lu ce texte \\
\hline
\end{tabular}

Quand on tente de rendre compte de cette contrainte dans un cadre DI/PL, on tombe immédiatement sur des difficultés. Dans une grammaire DI/PL, les règles d'ordre relient des paires de constituants. Pour exclure (34a), on a donc deux possibilités : soit on pose que les adverbes non-légers doivent précéder les adverbes légers; mais alors, on exclut également (34b). Soit les adverbes non-légers doivent suivre les verbes non-finis; mais dans ce cas, (34c) devrait être exclue. Le seul moyen de s'en sortir serait de postuler que fréquemment n'a pas exactement la même catégorie selon qu'il est à droite ou à gauche de l'auxiliaire ; or, rien ne semble soutenir une telle analyse.

Il apparaît donc que les grammaires DI/PL sont mal armées pour rendre compte du contraste $(34)$; il semble nécessaire de poser que, même si mieux et lu ne forment pas un 
constituant, ils appartiennent tous deux à un domaine dans lequel les adverbes nonlégers ne peuvent pas pénétrer. Si l'on revient sur la caractérisation des grammaires DI/ PL donnée en (4), le problème est donc lié à (4a) : il semble que le GV ne soit pas un domaine d'ordre libre. Afin de rendre compte de (34), il est donc préférable d'utiliser un cadre qui autorise un constituant à contenir des domaines opaques.

\subsection{Une analyse GAP}

La notion de position formalisée dans les GAP est un bon candidat pour modéliser le domaine opaque intervenant au sein du GV en français: on peut poser que les constituants légers (distincts de la tête) occupent tous une même position qui est interdite aux constituants non-légers.

Plus spécifiquement, on peut rendre compte de (34) en utilisant la grammaire suivante :

59 (35)

$a$.

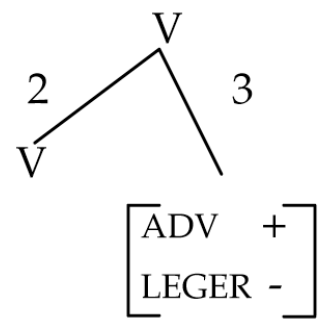

$b$.

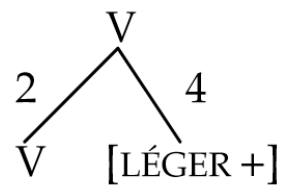

C.

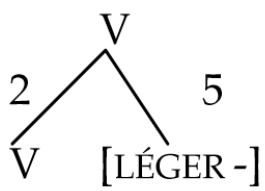
des adverbes non-légers; (35b) que la position 4 peut être occupée par n'importe quel constituant léger. Enfin, (35c) indique que la position 5 peut être occupée par n'importe quel constituant non-léger. l'adverbe non-léger fréquemment occupe la position 3, alors qu'il occupe la position (5) en (34b). Le participe et l'adverbe léger occupent tous deux la position 4, qui est la seule qui leur est autorisée. Enfin, le verbe tête occupe la position 2, en vertu d'une contrainte générale imposant à la tête d'être le pivot en français. (34a) est exclue, puisque fréquemment ne peut occuper que les positions 3 et 5 , alors que le participe et l'adverbe léger ne peuvent occuper que la position 4 .

(36)

$a$.

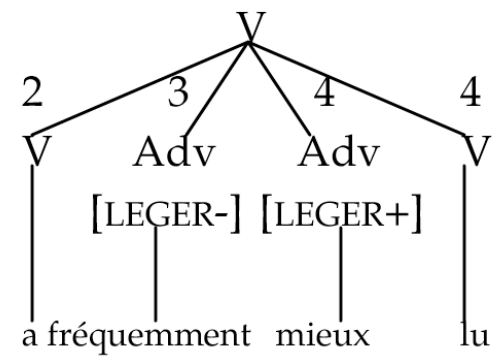

$b$.

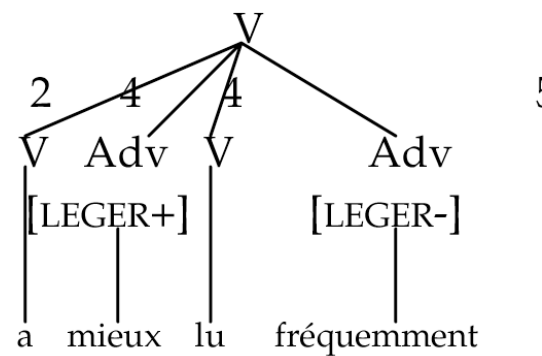

Les GAP permettent donc de rendre compte de l'observation qui posait problème pour l'approche DI/PL : en disant qu'il existe dans le GV une position réservée aux constituants 
légers, on obtient exactement l'effet qu'il était difficile d'obtenir dans une grammaire DI/ PL.

L'analyse proposée caractérise l'occupabilité des positions 3, 4 et 5 par leurs propriétés de poids : elle suppose que toute séquence léger/non-léger/léger est interdite. Cette analyse prédit donc que les phrases (37a) et (38a) ci-dessous sont agrammaticales. On constate effectivement un contraste, même s'il est moins net que le contraste observé en (34) ${ }^{12}$ :

\begin{tabular}{|l|l|l|}
\hline$(37)$ & a. & ? ? Paul a tout fréquemment lu \\
\hline & b. & Paul a tout lu fréquemment \\
\hline & c. & Paul a fréquemment tout lu \\
\hline$(38)$ & a. & ? ? Paul a rendu fréquemment hommage à Marie \\
\hline & b. & Paul a rendu hommage à Marie fréquemment \\
\hline & c. & Paul a fréquemment rendu hommage à Marie \\
\hline
\end{tabular}

\subsection{Une analyse GLD}

La formulation d'une analyse GLD de l'ordre dans le GV ne pose pas de problèmes particuliers. Il suffit de poser quatre champs topologiques distincts correspondant aux quatre positions de l'analyse GAP. Les domaines d'ordre des GV (34a) et (34b) seront respectivement :

(39)

$a$.

$b$.
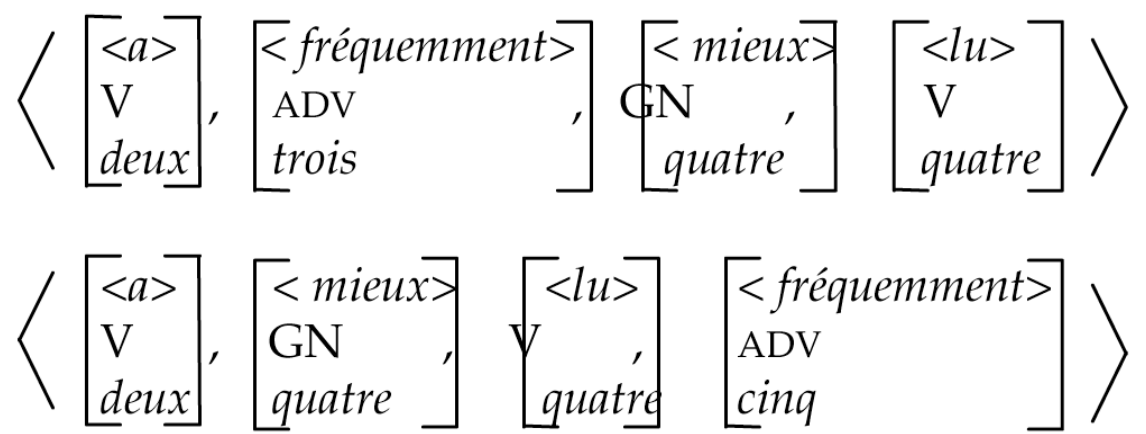

67 Les contraintes suivantes sur les valeurs de TOPO rendent compte du placement des adverbes :

\begin{tabular}{|l|l|l|}
\hline$(40)$ & a. & Un verbe fini est [TOPO deux]. \\
\hline & b. & Un objet léger est [TOPO deux] ou [TOPO quatre]. \\
\hline & c. & Un objet adverbial non-léger est [TOPO trois] ou [TOPO cinq]. \\
\hline
\end{tabular}




\begin{tabular}{|l|l|l|}
\hline & d. & Tout objet [TOPO trois] est adverbial. \\
\hline \hline & e. & Au plus un objet est [TOPO deux]. \\
\hline
\end{tabular}

\section{Deuxième exemple : l'ordre dans les phrases finies en allemand}

\subsection{Le problème}

\subsubsection{Les données de base}

L'ordre des mots dans les phrases allemandes pose un certain nombre de problèmes qui rendent les analyses en termes de grammaires syntagmatiques difficiles. Je me concentre ici sur l'analyse grossière des phrases à verbe conjugué contenant un auxiliaire.

Les cinq principaux types de phrases finies en allemand sont illustrés en (41) :

\begin{tabular}{|c|c|c|c|c|c|c|c|c|c|c|c|}
\hline (41) & a. & \multicolumn{4}{|c|}{ Jemand } & \multicolumn{2}{|c|}{ hat } & \multicolumn{2}{|l|}{ den Hund } & \multicolumn{2}{|l|}{ gefüttert } \\
\hline & & \multicolumn{4}{|c|}{ Quelqu'un } & \multicolumn{2}{|l|}{ a } & \multicolumn{2}{|l|}{ le chien } & \multicolumn{2}{|l|}{ nourri } \\
\hline & & \multicolumn{10}{|c|}{ Quelqu'un a nourri le chien } \\
\hline & b. & \multicolumn{4}{|c|}{ Wer } & \multicolumn{2}{|c|}{ hat } & \multicolumn{2}{|l|}{ den Hund } & gefüttert & ? \\
\hline & & \multicolumn{4}{|l|}{ Qui } & \multicolumn{2}{|l|}{ a } & \multicolumn{2}{|l|}{ le chien } & \multicolumn{2}{|l|}{ nourri } \\
\hline & & \multicolumn{10}{|c|}{ Qui a nourri le chien? } \\
\hline & c. & \multicolumn{3}{|l|}{ Hat } & \multicolumn{3}{|c|}{ jemand } & \multicolumn{2}{|l|}{ den Hund } & \multicolumn{2}{|l|}{ gefüttert } \\
\hline & & \multicolumn{3}{|l|}{ A } & \multicolumn{3}{|c|}{ quelqu'un } & \multicolumn{2}{|l|}{ le chien } & \multicolumn{2}{|l|}{ nourri } \\
\hline & & \multicolumn{10}{|c|}{ Quelqu'un a-t-il nourri le chien? } \\
\hline & d. & \multicolumn{2}{|c|}{ daß } & \multicolumn{4}{|c|}{ jemand } & \multicolumn{2}{|l|}{ den Hund } & gefüttert & hat \\
\hline & & \multicolumn{2}{|l|}{ que } & \multicolumn{4}{|c|}{ quelqu'un } & \multicolumn{2}{|l|}{ le chien } & nourri & a \\
\hline & & \multicolumn{10}{|c|}{ que quelqu'un a nourri le chien } \\
\hline & e. & wer & den & Iund & & & gefüttert & & hat & & \\
\hline & & qui & le c & & & & nourri & & a & & \\
\hline & & qui & & le & & & & & & & \\
\hline
\end{tabular}


70

(41a-c) sont des principales, alors que (41d-e) sont des subordonnées. Dans les principales, le verbe conjugué apparaît en première ou en deuxième position dans la phrase: dans les déclaratives (41a) et les questions qu- (41b), le verbe conjugué est précédé par un de ses dépendants ${ }^{13}$, qui doit être le constituant qu-dans le cas d'une question (41b). Les autres dépendants sont réalisés entre le verbe conjugué et le participe ${ }^{14}$. Dans une question polaire principale (41c), rien ne précède le verbe conjugué, et tous les dépendants sont réalisés entre les deux verbes.

71 Dans les subordonnées, les deux verbes apparaissent en fin de phrase, après leurs dépendants. La tête de phrase est occupée, suivant les cas, par un complémenteur (relatives à complémenteur ou complétives, cf. 41d), ou par un syntagme qu- (relatives qu, questions qu- enchâssées).

\subsubsection{L'analyse traditionnelle}

72 La grammaire traditionnelle de l'allemand rend compte de cette distribution en supposant qu'une phrase est divisée en un certain nombre de champs topologiques. Une phrase commence (optionnellement) par un champ avant (Vorfeld, vf), qui contient au plus un dépendant. Puis vient la frontière gauche (linke Satzklammer, ls), qui contient un et un seul constituant. Le champ central (Mittelfeld, $m f$ ) contient les autres dépendants, et la phrase se termine par un complexe verbal ou frontière droite (rechte Satzklammer, rs) contenant un nombre variable de verbes ${ }^{15}$. (42) reprend l'analyse des phrases de (41) en termes de champs topologiques :

\begin{tabular}{|l|l|l|l|l|l|l|l|}
\hline (42) & & vf & ls & mf & \multicolumn{2}{l|}{ rs } \\
\hline & a. & Jemand & hat & & den Hund & & gefüttert \\
\hline & b. & Wer & hat & & den Hund & & gefüttert \\
\hline & c. & & Hat & jemand & den Hund & & gefüttert \\
\hline & d. & & daß & jemand & den Hund & gefüttert & hat \\
\hline & e. & & wer & & den Hund & gefüttert & hat \\
\hline
\end{tabular}

Cette analyse rend compte de la différence de distribution des verbes finis dans les principales et les subordonnées en posant que le verbe fini est en compétition avec le complémenteur (41d) ou le syntagme qu- (41e) pour l'occupation du champ topologique $l s$ : ls ne pouvant être occupé par plus d'un constituant, le verbe fini ne peut pas s'y trouver dans une subordonnée; mais ls devant obligatoirement contenir un constituant, le verbe fini doit s'y trouver dans une principale.

\subsubsection{Justification du champ /s}

74 L'analyse traditionnelle repose donc crucialement sur l'idée qu'il existe une position spécifique de frontière gauche qui contient toujours un et un seul constituant; suivant les cas, un verbe, un complémenteur, ou un syntagme qu-. Le recours à l'idée d'une position 
unique est justifié, non seulement par la distribution des verbes conjugués, mais également par un certain nombre de propriétés qui montrent que trois unités de catégories hétérogènes doivent former une classe distributionnelle. Je reprends ici deux des arguments cités par Kathol \& Pollard (1995) :

75 (a) Les constituants occupant la frontière gauche ont tous le même statut vis à vis de l'interprétation du marquage accentuel. Le marquage accentuel du constituant occupant la frontière gauche est interprété comme une focalisation de la valeur de vérité de la proposition exprimée par la phrase (verum focus) ${ }^{16}$ :

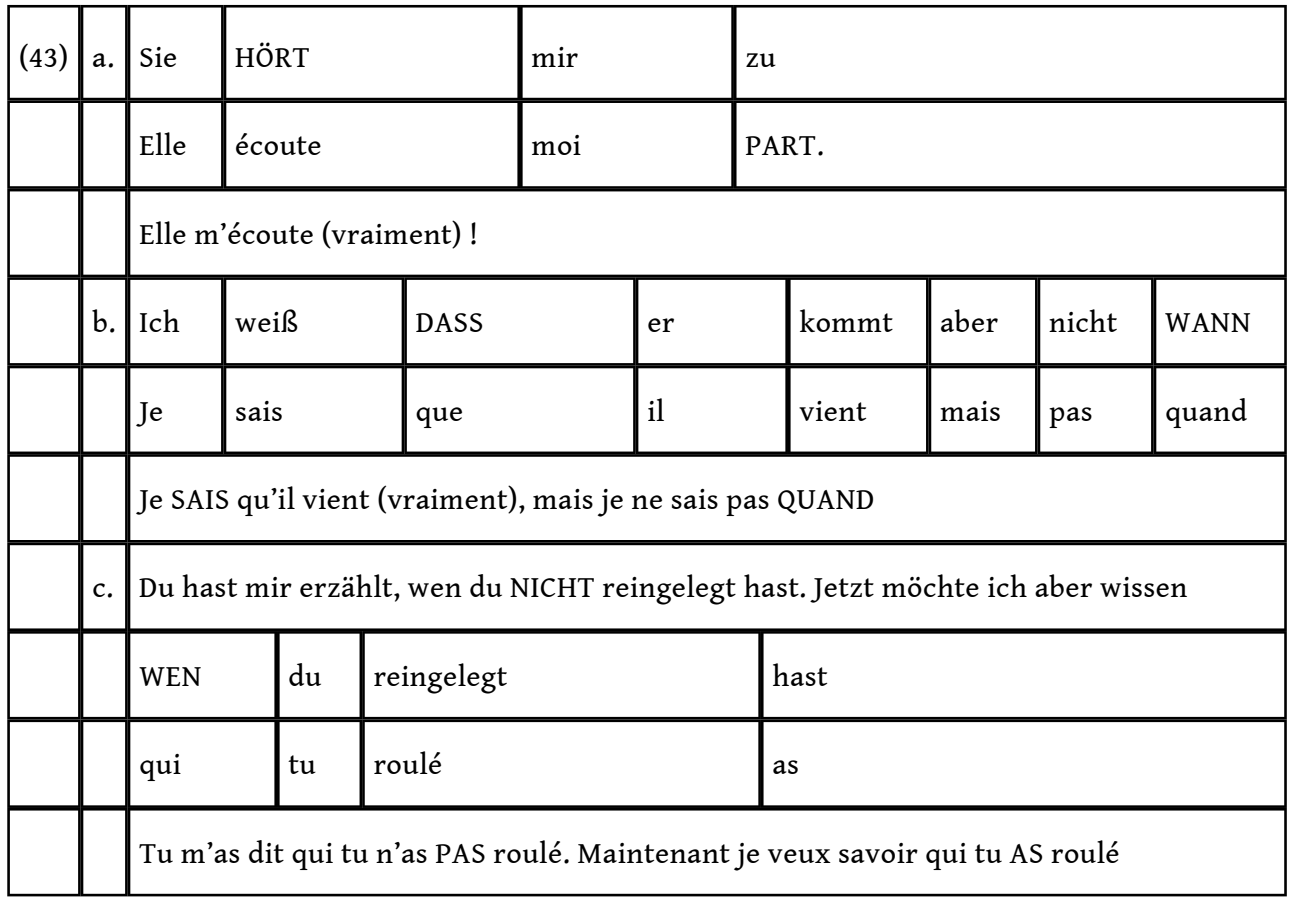

76 Les constituants en (43a-c) n'ayant pas la même catégorie ni la même fonction, le seul moyen de rendre compte du fait qu'ils forment une classe naturelle pour l'interprétation de l'accentuation est de dire qu'ils occupent une même position.

(b) Dans certains dialectes, quand le sujet d'une phrase enchâssée est de deuxième personne, celui-ci peut être réalisé non pas comme un pronom, mais comme un affixe syntagmatique $^{17}$ dont la forme ressemble à celle de la flexion verbale de deuxième personne :

\begin{tabular}{|l|l|l|l|}
\hline$(44)$ & \multicolumn{2}{|l|}{ Ich weiß } \\
\hline \hline & \multicolumn{2}{|l|}{ Je sais } \\
\hline & a. & daß-ste & kommst \\
\hline & & que+2SG & viens \\
\hline & & que tu viens \\
\hline
\end{tabular}




\begin{tabular}{|l|l|l|l|l|l|}
\hline & b. & mit & wem-ste & weggegangen & bist \\
\hline \hline & & avec & qui+2SG & parti & es \\
\hline & & avec qui tu es parti & & \\
\hline
\end{tabular}

De manière intéressante, la réalisation du sujet comme un affixe est également possible dans une principale ; dans ce cas, un sujet pronominal plein est impossible :

\begin{tabular}{|l|l|l|l|l|}
\hline (45) & a. & Kommst & du & endlich ? \\
\hline & & Viens & tu & finalement \\
\hline & & Tu viens, finalement? \\
\hline & b. & Komm-ste & endlich? \\
\hline & & Viens+2SG & finalement \\
\hline & & Tu viens, finalement? \\
\hline & c. & * Komm-ste & du & endlich? \\
\hline & & Viens+2SG & tu & finalement \\
\hline
\end{tabular}

79 Ici encore, le seul moyen d'identifier le site sur lequel le sujet affixal est réalisé est de supposer que les trois constituants occupent une même position.

\subsection{Une tentative d'analyse DI/PL}

Pour formuler une analyse DI/PL de la phrase allemande, de nombreuses options sont disponibles ${ }^{18}$. J'examine dans ce paragraphe une tentative de transcription directe de l'analyse traditionnelle en termes de champs dans un cadre DI/PL.

81 L'analyse en termes de champs ne faisant intervenir aucune organisation hiérarchique, l'hypothèse la plus simple est de poser que la phrase est un constituant entièrement plat. Une telle hypothèse revient à proposer les structures suivantes pour les phrases (41a) et (41d) :

82 (46) 
a.

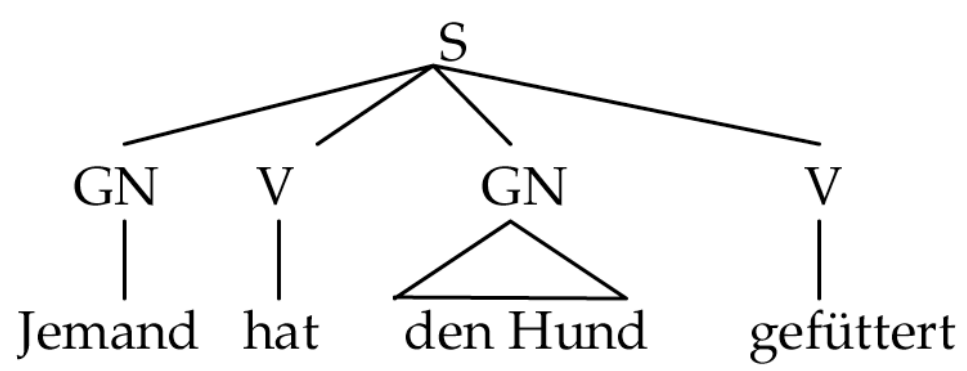

b.

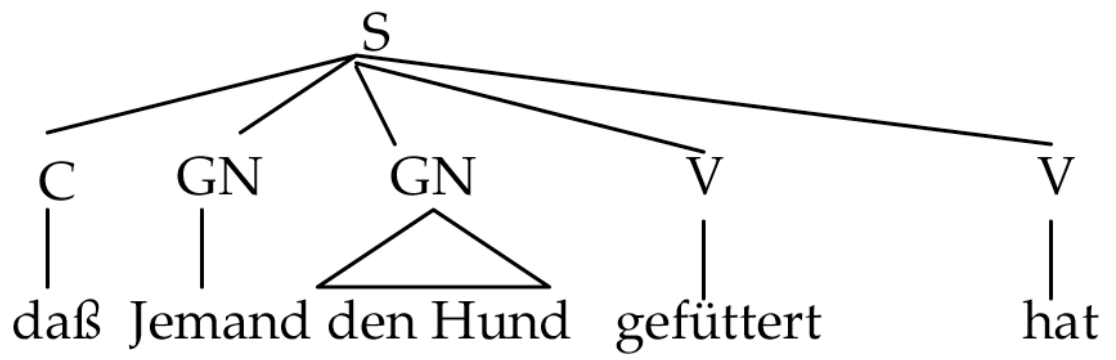

L'analyse proposée pour (41a) est possible, si on admet qu'un mécanisme d'attraction d'arguments assure que l'auxiliaire hat hérite des dépendants de gefüttert ${ }^{19}$. De même, on peut arriver à l'analyse proposée pour (41d) en admettant que le complémenteur daß est tête de la phrase, et hérite des dépendants de hat. Proposer une structure plate pour (41e) est plus problématique, dans la mesure où le syntagme qu-ne peut être analysé ni comme la tête de la phrase ni comme un dépendant de la tête, mais doit être analysé comme un filler. Il faut donc proposer un schéma de dominance tête-dépendants-filler, qui permet de combiner les trois types d'unités au même niveau.

L'hypothèse d'une structure plate de la phrase allemande est donc soutenable, à défaut d'être attrayante. En revanche, on tombe sur une difficulté quand on tente de rendre compte des données présentées en 3.1.3. Ces observations montrent qu'il est nécessaire de disposer d'un moyen d'identifier le constituant occupant la frontière gauche de la phrase, au sens de l'analyse traditionnelle. Supposons que cette identification prend la forme du marquage de ce constituant comme portant un certain trait $[\mathrm{FG}+]$. Ce constituant $[\mathrm{FG}+]$ ne peut être identifié ni par sa catégorie syntaxique, ni par sa fonction grammaticale. La seule option disponible est donc d'identifier ce constituant par sa position linéaire au sein d'un syntagme. Or, on constate que le constituant $[\mathrm{FG}+]$ n'apparaît pas toujours dans la même position linéaire dans la phrase : par exemple, il est premier dans les subordonnées, alors qu'il est en seconde position dans les déclaratives et les questions qu- principales. Poser une structure plate pour l'ensemble des types de phrases ne pourra donc pas donner le résultat escompté : même si on peut formuler des règles ordonnant adéquatement les constituants, on ne pourra pas déterminer quel est le constituant marqué $[\mathrm{FG}+]^{20}$.

L'analyse de la phrase allemande pose donc un problème pour les grammaires DI/PL: même s'il est possible d'obtenir l'ordre des mots adéquat, il manque un moyen d'identifier une certaine position qui joue un rôle distingué. 


\subsection{Une analyse GLD}

Kathol (1995) propose une analyse GLD des données exposées en 3.1. Les GLD ont deux caractéristiques qui facilitent l'analyse des données présentées en 3.1 :

87 (47a) a pour conséquence que la frontière gauche est clairement identifiable : il suffit de supposer que le marquage $[\mathrm{FG}+]$ est sensible à la valeur de TOPO. Si un seul objet peut recevoir la valeur $l s$ pour TOPO dans une phrase, il suffit de dire qu'un objet est [TOPO ls] si et seulement si il est [FG+]; les phénomènes affectant la frontière gauche affecteront cet objet et cet objet seul. (47b) a de plus l'avantage de faciliter l'analyse de (41e) : il n'est pas nécessaire de poser que le filler, la tête et ses dépendants sont combinés en une seule étape. Spécifiquement, Kathol (1995) propose la structure de composition suivante pour (41e) :

$88 \quad(48)$

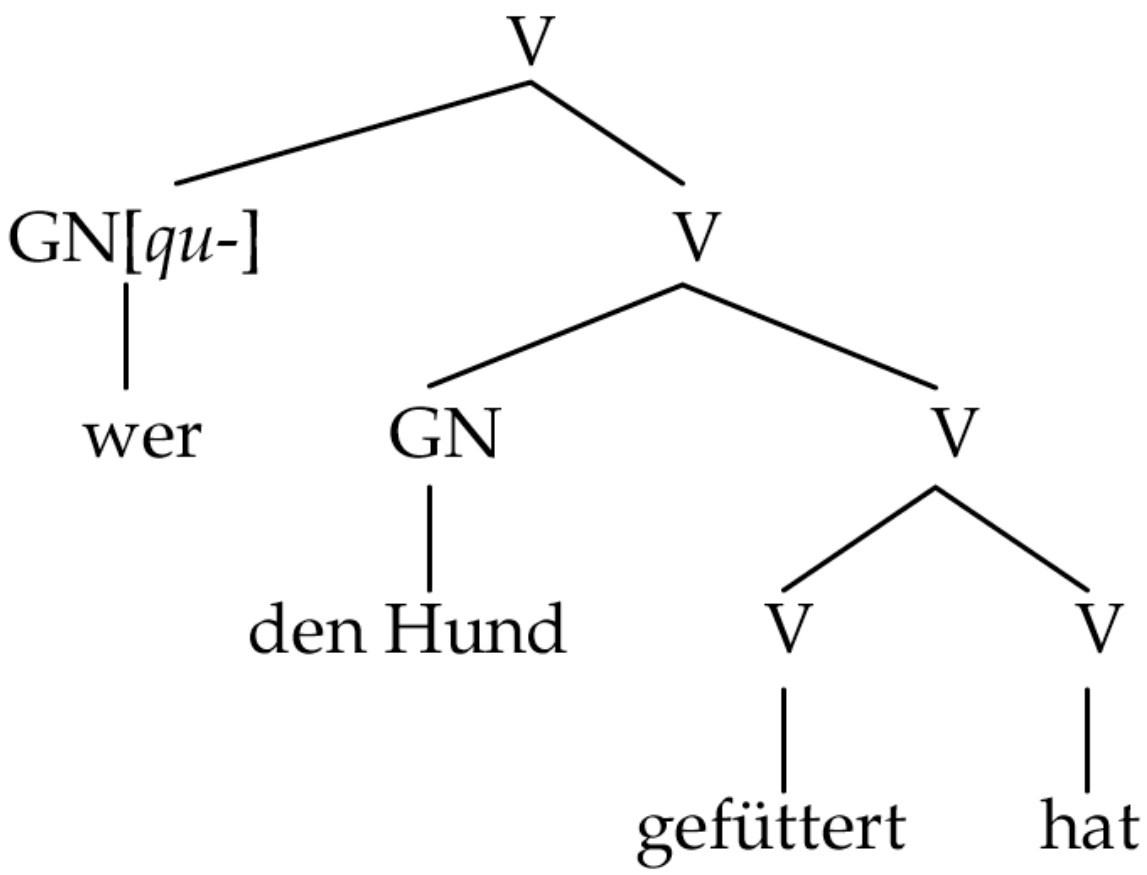

89 Les domaines d'ordre associés par Kathol aux phrases (41a), (41d) et (41e) sont donnés en (49) : 
a.

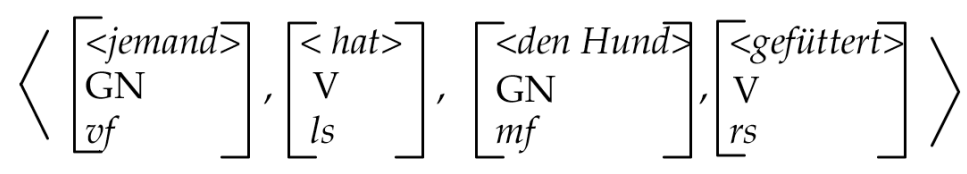

b.

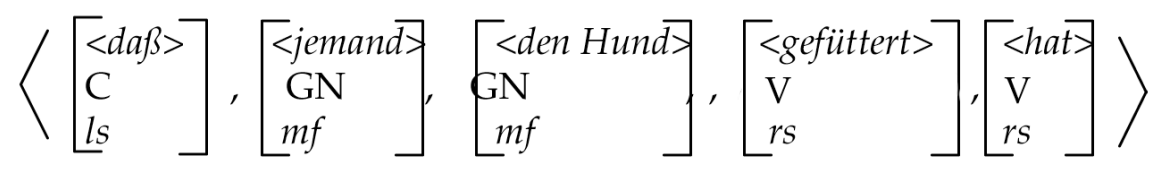

$c$.

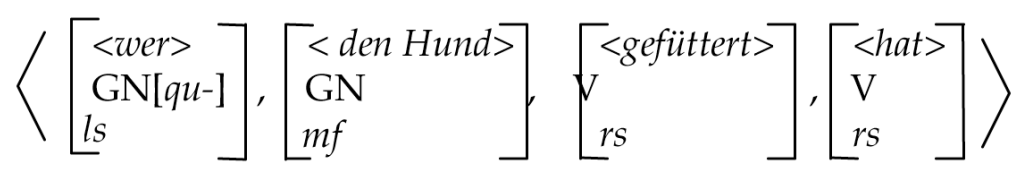

91 Les contraintes grammaticales utilisées pour obtenir cette assignation de champs topologiques sont résumées, de manière informelle et simplifiée, en (50).

\begin{tabular}{|l|l|l|}
\hline (50) & a. & Dans un domaine d'ordre, au plus un objet est marqué [TOPO ls]. \\
\hline & b. & Dans une phrase finie, au moins un constituant est marqué [TOPO ls]. \\
\hline & c. & Un verbe a pour valeur de TOPO soit ls soit rs. \\
\hline & d. & Un complémenteur a pour valeur de TOPO ls. \\
\hline & e. & Un syntagme qu- a pour valeur de TOPO soit vf soit ls. \\
\hline & f. & Dans une principale finie, le verbe tête a pour valeur de TOPO ls. \\
\hline & g. & $\begin{array}{l}\text { Dans une subordonnée finie, soit un complémenteur soit un syntagme qu- a pour } \\
\text { valeur de TOPO ls. }\end{array}$ \\
\hline
\end{tabular}

92 (41a) étant une principale, le verbe tête occupe la position ls (50f). ls ne pouvant pas accueillir plus d'un constituant (50a), le second verbe est nécessairement dans rs (50c). (41d) contenant un complémenteur, celui-ci occupe la position ls (50d). Les deux verbes se retrouvent donc nécessairement dans $r s(50 \mathrm{a}-\mathrm{c})$. Enfin, (41e) étant une subordonnée ne contenant pas de complémenteur, ls doit contenir le syntagme qu-, en vertu de (50g).

\subsection{Une analyse GAP}

Les champs topologiques dans une phrase finie en allemand jouent le même rôle que les positions dans une GAP : ils délimitent, à l'intérieur d'un syntagme, un certain nombre de domaines d'ordre opaques strictement ordonnés entre eux. On va donc formaliser les champs topologiques comme des positions. Le champ ls étant le seul champ qui contient toujours exactement un élément, c'est le seul candidat possible pour jouer le rôle de pivot ${ }^{21}$. En supposant que la couleur pivot est 2, une analyse plausible de (41a-d-e) est donnée en $(51)^{22}$ : 
a.

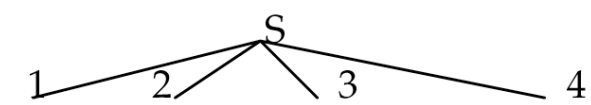

GN

Jemand hat den Hund gefüttert

b.

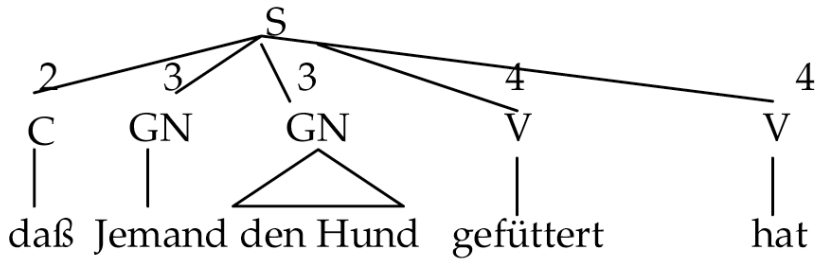

C.

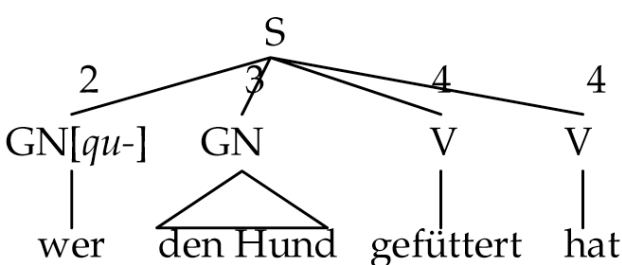
structure de composition des arbres (51b) et (51c). Dans ces deux cas, le pivot a une catégorie différente de celle du syntagme. En GAP, il n'est pas possible d'arriver à ce résultat en utilisant une structure de composition analogue à (48). On est contraint à poser la structure de composition (52) pour (51c) :

$97 \quad(52)$

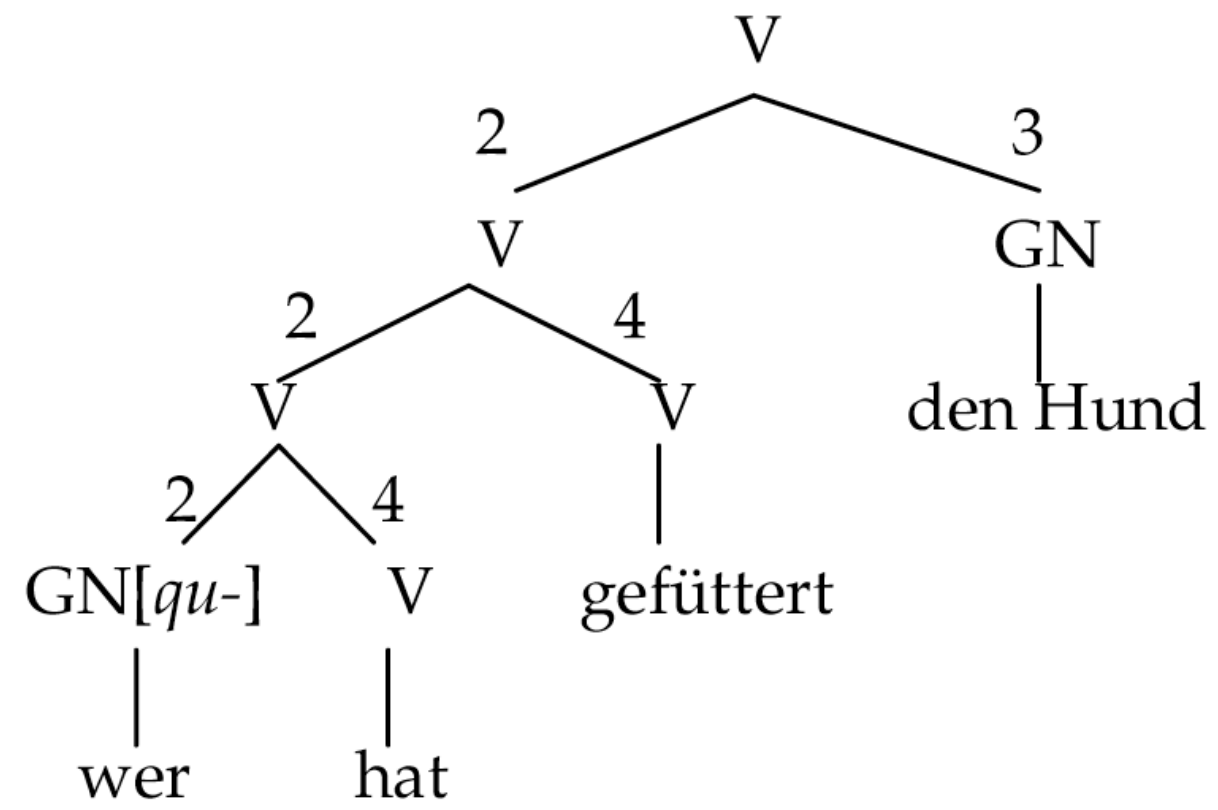


Cette structure de composition est très éloignée de celles qui sont supposées habituellement, et a la forme inverse de la structure utilisée par Kathol. Cependant, elle ne pose pas de problèmes particuliers ${ }^{23}$.

Il est intéressant de noter que rien dans l'analyse GLD n'interdit l'utilisation d'une structure de composition semblable à (52) : l'assignation des champs topologiques étant totalement indépendante des schémas de composition dans une GLD, plusieurs structures de composition sont systématiquement envisageables pour une même phrase. Par contraste, les GAP supposent une association entre les schémas de composition (arbres élémentaires) et les positions, contraignant en particulier chaque arbre élémentaire à contenir un pivot. En conséquence, les structures de compositions envisageables sont contraintes par les linéarisations possibles.

00 L'exemple de la représentation des champs topologiques montre donc que les GAP sont plus contraintes que les GLD: le rôle des pivots limite le nombre de structures de composition qui peuvent être envisagées pour un syntagme donné.

\section{Conclusion}

1 Les analyses présentées dans les sections 2 et 3 fournissent des arguments pour différencier les grammaires DI/PL des GAP et des GLD; dans le cas du GV en français comme dans celui de la phrase en allemand, il est plausible de concevoir un type de constituant comme une séquence strictement ordonnée de domaines opaques pour l'ordre. Alors que les grammaires DI/PL rencontrent des difficultés pour modéliser une telle conception de l'ordre, les GAP comme les GLD sont spécifiquement conçues pour la formaliser.

2 La différence entre GAP et GLD est plus difficile à mettre au jour.On a vu que l'on pouvait formuler la même analyse des GV du français dans les deux cadres; pour l'analyse de la phrase allemande, il est apparu que les GAP étaient plus contraintes que les GLD : le rôle des pivots dans les GAP contraint les structures de composition qui peuvent être envisagées. L'absence d'une notion de pivot en GLD laisse plus de possibilités ouvertes. En ce sens, les GAP sont plus contraintes que les GLD.

103 Cette différence étant notée, on peut se demander si le fait de poser que tout syntagme contient un pivot est justifié. Posée en termes généraux, cette question ne peut être tranchée qu'après une analyse détaillée des types de syntagmes d'une langue, voire des langues en général, tâche qui ne peut évidemment pas être entamée ici. On doit se demander, d'une part, pour chaque type de syntagme d'une langue, s'il contient un pivot, et d'autre part, si toutes les langues ont des types de syntagmes à pivot.

04 Il y a une autre différence essentielle entre GAP et GLD, que je ne ferai que mentionner. Dans certaines constructions, la composition et la linéarisation semblent être totalement découplées ${ }^{24}$. Pour prendre un exemple simple, il est plausible de supposer que les phrases (53) aient la même structure de composition:

\begin{tabular}{|l|l|l|}
\hline (53) & a. & Un homme qui portait un chapeau est entré \\
\hline \hline & b. & Un homme est entré qui portait un chapeau \\
\hline
\end{tabular}


La relative qui est linéarisée adjacente au nom qu'elle modifie en (53a) est extraposée en fin de phrase en (53b). Au premier abord, rien ne semble indiquer que la structure de composition des deux phrases soit différente (la relative a la même relation grammaticale au nom dans les deux cas); on peut donc supposer que la seule différence entre les deux phrases est une différence de linéarisation.

Cette hypothèse peut être formalisée dans le cadre des GLD : les constituants n'étant pas des domaines opaques pour l'ordre, il suffit de poser que la relative est insérée dans le domaine d'ordre de la phrase en (53b), et pas en (53a) ${ }^{25}$. En revanche, elle ne peut pas être maintenue dans le cadre des GAP, où les constituants sont des domaines opaques pour l'ordre, et où seule la linéarisation de constituants dépendant d'un même pivot est susceptible de varier.

\section{Annexe : GAP-STT}

GAP-STT (Grammaires d'Arbres Polychromes sous formes de Structures de Traits Typées) est une reconstruction des GAP dans le formalisme de structures de traits typées utilisé en HPSG. Dans ce formalisme, les objets linguistiques (mots, syntagmes, etc.) sont analysés comme des types associés à des structures de traits complexes, chaque trait prenant pour valeur un nouvel objet linguistique typé. La grammaire (entrées lexicales, règles, principes, etc.) est constituée d'un ensemble de contraintes sur les objets linguistiques.

En GAP-STT, le type d'objet linguistique de base est le nœud. Chaque nœud est associé à une catégorie syntaxique. Dans les GAP classiques, la catégorie est une valeur atomique ${ }^{26}$. Afin de disposer des outils d'analyse de HPSG, on remplace ici cette valeur atomique par l'attribut SYNSEM. La valeur de SYNSEM a la structure habituellement supposée en HPSG, distinguant catégorie et contenu, traits de tête et traits de valence, etc.

Le type noud a deux sous-types: noud-lex(ical) et arbre. Un arbre porte deux traits supplémentaires, qui encodent sa structure syntagmatique. BRANCHES encode sa structure syntagmatique " compactée », alors que BR-LOC (branches locales) est un trait auxiliaire qui sert à distinguer, parmi les branches de l'arbre, celles qui ont été introduites au niveau de ce nœud.

$110(54)$

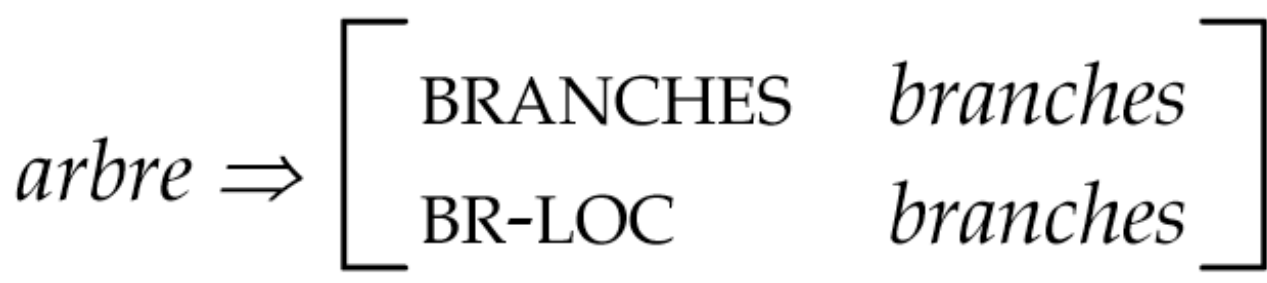

BRANCHES et BR-LOC prennent tous deux comme valeur une liste de branches, où une branche est constituée d'une couleur et d'un nœud : 


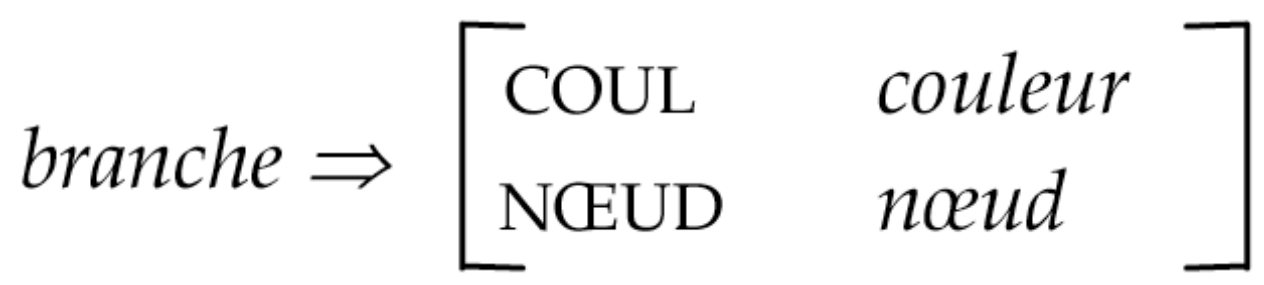

On admet pour les besoins de l'exposé qu'il y a cinq couleurs numérotées de 1 à 5 , et que la couleur du pivot est 2. Il reste à contraindre la liste des branches à respecter les contraintes sur les GAP : les couleurs doivent apparaître dans l'ordre, et le pivot est obligatoirement instancié. Ce qui est assuré par la contrainte suivante :

114 (56)

$$
\begin{aligned}
& \text { liste }\left(\left[\begin{array}{c}
\text { branche } \\
\text { COUL 1 }
\end{array}\right]\right) \oplus\left\langle\left[\begin{array}{l}
\text { branche } \\
\text { coul 2 }
\end{array}\right]\right\rangle \oplus \text { liste }\left(\left[\begin{array}{c}
\text { branche } \\
\text { COUL 3 }
\end{array}\right]\right) \oplus \\
& \text { liste }\left(\left[\begin{array}{c}
\text { branche } \\
\text { coul } 4
\end{array}\right]\right) \oplus \quad\left(\left[\begin{array}{ll}
\text { branche } \\
\text { Coul } & 5
\end{array}\right]\right)
\end{aligned}
$$

115 La grammaire à proprement parler prend la forme d'une disjonction de contraintes sur les nœuds syntagmatiques. Spécifiquement, l'équivalent d'un arbre élémentaire est une contrainte reliant le SYNSEM d'un arbre et la valeur de son attribut BR-LOC. L'exemple suivant est le correspondant de l'arbre élémentaire (35a) :

116 (57)

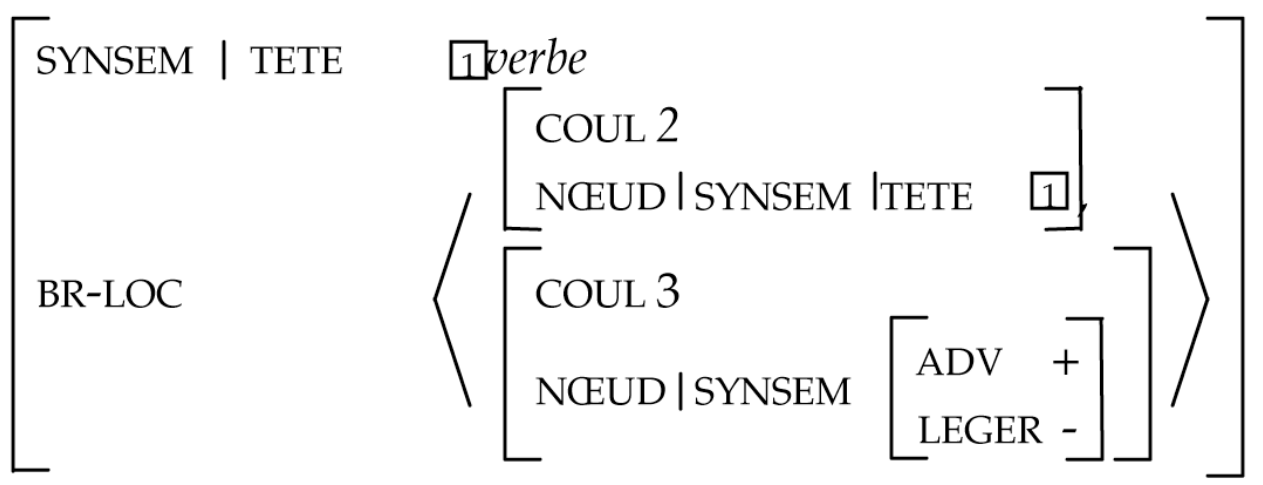

117 Il reste à spécifier le principe de compactage, qui indique la relation entre BRANCHES et BR-LOC. Dans la mesure où j'ai remplacé les catégories atomiques des GAP par les synsem de HPSG, la question se pose de savoir sur quel attribut tester l'identité de catégorie mise en jeu dans le compactage. Le candidat le plus naturel est le trait TETE, qui regroupe toutes les informations dont la valeur est partagée entre un syntagme et sa tête ${ }^{27}$.

Le principe de compactage peut alors être formulé comme en (58) :

(58) Principe de compactage 


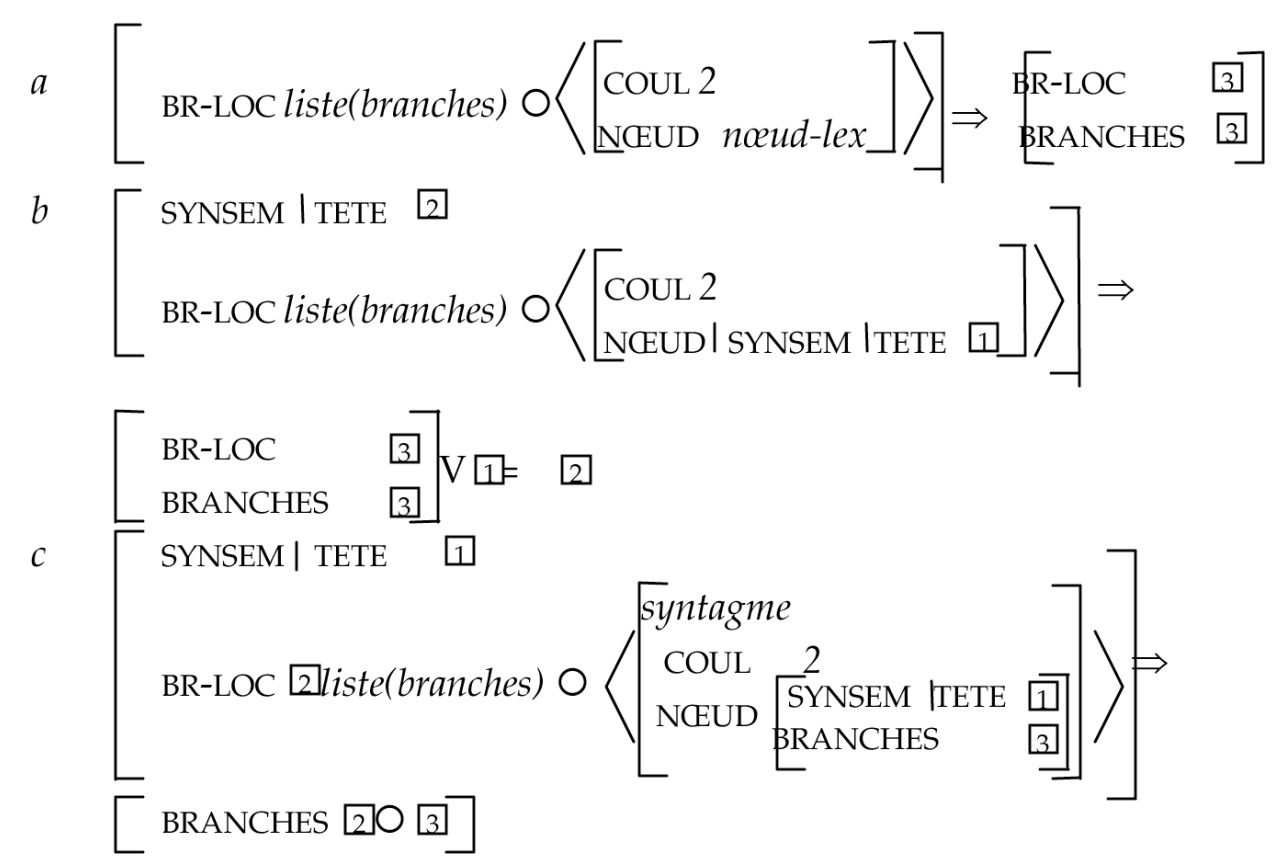

Comme dans la formulation classique de GAP, trois cas sont distingués : (i) soit le pivot de l'arbre local est lexical (type nœeud-lex), et la valeur des deux attributs est identique ; (ii) soit la mère et le pivot ont des catégories différentes, et, là encore, la valeur des deux attributs est identique; (iii) enfin, si la mère et le pivot sont de même catégorie, et que le pivot est syntagmatique, alors la structure syntagmatique est obtenue en mélangeant la liste des branches locales de la mère avec la liste des branches du pivot. La définition de branches en (56) fera alors en sorte que les différentes couleurs se retrouvent dans leur ordre ordinaire.

Il y a un point sur lequel la reconstruction des GAP qui vient d'être proposée est infidèle : dans les GAP, l'ordre entre les branches d'une même couleur est déterminé par l'ordre dans lequel les arbres élémentaires ont été composés. En GAP-STT, l'ordre entre les branches d'une même couleur n'est pas contraint par la composition. Je pose donc que l'ordre entre les branches portant une même couleur sera éventuellement contraint par des règles d'ordre indépendantes.

\section{BIBLIOGRAPHIE}

ABEILLE, A. \& GODARD, D. (1996) La complémentation des auxiliaires en français, Langages 122, pp. 32-61.

ABEILLE, A. \& GODARD, D. (à paraître) French word order and lexical weight, in R. Borsley, Syntactic categories, Academic Press, New York. 
ABEILLE, A. \& GODARD, D. (1998) A lexical account of quantifier floating, inG. Webelhuth, J.-P. Koenig et A. Kathol, Lexical And Constructional Aspects of LinguisticExplanation, CSLI Publications, Stanford.

BONAMI, O. \& GODARD D. (à paraître) Inversion du sujet, constituance et ordre des mots, in G. Lardreau, Cahier Jean-Claude Milner, Verdier, Paris.

BONAMI, O., GODARD, D. \& MARANDIN, J.-M. (1998) French subject inversion in extraction contexts, in G. Bouma, G.-J. Kruijff et R.T. Oehrle, Proceedings of the joint conference on Formal Grammar, Head-driven Phrase Structure Grammar, and Categorial Grammar, DFKI, Saarbrücken.

CORI, M. \& MARANDIN, J.-M. (1993) Grammaires d'Arbres Polychromes, TAL 34.1, pp. 101-132. DOWTY, D. (1997) Towards a minimalist theory of syntactic structure, in Hock et Sitjma, Discontinuous constituency, Mouton de Gruyter, La Haye.

GAZDAR, G., KLEIN, E., PULLUM, G. K. \& SAG, I. A. (1985) Generalized Phrase Structure Grammar, Blackwell, Londres.

HINRICHS, E. \& NAKAZAWA, T. (1994) Linearizing AUXs in German verbal complexes, in Nerbonne et al. (1994).

KATHOL, A. (1995) Linearization-based German syntax, PhD dissertation, Ohio State University.

KATHOL, A. \& POLLARD, C. (1995) On the left periphery of German subordinate clauses, in Proceedings of WCCFL 14, CSLI Publications, Stanford.

MILLER, P. (1992) Clitics and constituents in Phrase Structure Grammar, Garland, New York.

NERBONNE, J., NETTER, K. \& POLLARD, C. (1994) German in Head-Driven Phrase Structure Grammar, CSLI Publications, Stanford.

POLLARD, C. \& SAG, I.A. (1987) Information-based syntax and semantics, CSLI Publications, Stanford.

POLLARD, C. \& SAG, I.A. (1994) Head-driven Phrase Structure Grammar, CSLI Publications, Stanford et The University of Chicago Press, Chicago.

REAPE, M. (1994) Domain union and word-order variation in German, in Nerbonne et al. (1994).

SAG, I. A. (1997) English relative clause constructions, Journal of linguistics 33, pp. 431-484.

USZKOREIT, H. (1987) Word Order and Constituent Structure in German, CSLI Publications, Stanford.

\section{NOTES}

*. Je remercie K. Baschung, G. Boyé, P. Cabredo-Hofherr, D. Godard, et, tout spécialement, J.-M. Marandin, pour leurs commentaires et suggestions.

1. Par composition, on entend la structure spécifiant les composants d'une unité complexe et les relations grammaticales que ces composants entretiennent.

2. Rien n'impose que le pivot soit occupé par la tête syntaxique du syntagme ; par exemple, selon les analyses proposées ici, le pivot de GV est sa tête en français, mais le pivot de S n'est pas nécessairement la tête en allemand.

3. Comme en HPSG, je suppose qu'un syntagme dont la tête est un verbe est lui aussi de catégorie verbe; un GV est une unité de catégorie verbe dont la liste de compléments est vide; de même pour les phrases : ce sont des verbes dont la liste des sujets est vide.

4. La théorie de Kathol est en partie inspirée des travaux de Dowty (1997) et Reape (1994), dont elle partage plusieurs caractéristiques. L'encodage de la notion de champ topologique, 
traditionnelle en syntaxe des langues germaniques, est cependant particulière à l'approche de Kathol. Cette caractéristique est cruciale pour rendre compte des données examinées dans les sections 2 et 3. Cf. Bonami \& Godard (à paraître), Bonami et al. (1998) pour une application de la théorie de Reape au traitement de l'inversion du sujet en français.

5. Ou composition d'arguments (argument composition). Je choisis le terme d'attraction pour éviter toute confusion avec la composition au sens défini en introduction.

6. Le symbole $\oplus$ note la concaténation de listes.

7. Le symbole $\bigcirc$ note l'opération non-déterministe de mélange (shuffle) de listes; $A \bigcirc B$ peut décrire toute liste contenant tous les éléments de $A$ et de $B$, et où l'ordre entre les éléments de $A$ d'une part, et les éléments de $B$ d'autre part, est respecté.

8. Abeillé \& Godard utilisent un trait WEIGHT (poids) qui peut prendre au moins deux valeurs : lite (Léger) ou nonlite (non-léger). Elles laissent ouverte la possibilité qu'une troisième valeur heavy ( lourd) soit appropriée pour ce trait. J'utilise un trait binaire pour simplifier.

9. Je laisse de côté le problème de l'ordre entre les adverbes légers et les autres constituants légers.

10. Je ne tiens pas compte ici des incises, et je suppose que (32a) n'est possible qu'avec l'intonation spécifique qui en est caractéristique.

11. La spécification [ADV-] en (33c) sert à ordonner correctement les quantifieurs nus par rapport aux autres constituants légers.

12. (37a) est marginalement acceptable si tout est accentué, alors que tout n'est pas accentué par défaut en (37b-c). Abeillé \& Godard notent, de la même manière, que les adverbes légers peuvent être linéarisés à droite du GV quand ils sont accentués (cf. Paul a lu ce texte BIEN). On peut rendre compte de ces données en posant qu'un constituant léger peut devenir non-léger quand il est accentué ; sous cette analyse, (37a) sera acceptable si tout et fréquemment occupent la position 3 , et non la position 4. Quoi qu'il en soit, la meilleure acceptabilité de (37a-38a) ne remet pas en cause le fait qu'une notion de domaine opaque à l'intérieur d'un constituant est nécessaire pour rendre compte de (34); il se peut simplement que la caractérisation de l'occupabilité des positions 3, 4 et 5 en purs termes de poids soit insuffisante.

13. Par dépendants d'un verbe, on entend son sujet, ses compléments, ainsi que les ajouts qui prennent comme argument le verbe ou une de ses projections.

14. Ce tableau de la syntaxe de l'allemand est évidemment largement simplifié; en particulier, on a délibérément ignoré les phénomènes d'extraposition, qui permettent à un dépendant d'un verbe ou d'un nom d'être réalisé à droite des verbes.

15. On ignore ici encore le champ arrière (Nachfeld), qui accueille les constituants extraposés.

16. Il est important de noter que l'on peut également accentuer un verbe en position finale, mais que cette accentuation ne reçoit pas alors la même interprétation (elle est interprétée comme une focalisation de ce verbe) :

nicht zuSIEHT)

pas)

17. Un affixe syntagmatique est un affixe se réalisant sur une des frontières d'un constituant, quelle que soit l'identité lexicale du mot occupant cette frontière; cf. Miller (1992). Le statut syntagmatique de l'affixe de deuxième personne n'est décelable que quand la frontière gauche est occupée par un syntagme qu-, l'occupant de la frontière gauche est constitué d'un seul mot dans les autres cas.

18. Voir les articles réunis dans Nerbonne et al. (1994) pour un panorama des possibilités dans le cadre de HPSG.

19. L'hypothèse selon laquelle l'auxiliaire hérite des dépendants de l'auxilié est justifiée indépendamment par l'ordre des mots dans le complexe verbal; cf. Hinrichs \& Nakazawa (1994).

20. Une structure plus hiérarchisée dans l'esprit de Uszkoreit (1987) tombe sur des difficultés analogues: la structure des phrases n'étant pas plate, le problème n'est plus de savoir quel 
constituant immédiat d'un syntagme est [FG +] (c'est toujours le premier), mais quels sont les syntagmes qui contiennent une frontière gauche.

21. On utilise ici crucialement le fait que le pivot d'un syntagme n'est pas nécessairement sa tête; spécifiquement, dans les subordonnées, le pivot n'est jamais la tête.

22. Faute de place, je ne peux détailler ici la grammaire permettant d'obtenir (51).

23. Si on importe dans le formalisme des GAP l'analyse standard des marqueurs en HPSG, une phrase dont le verbe tête est combiné avec un complémenteur sera considérée comme une phrase marquée par ce complémenteur; rien n'impose que le complémenteur soit combiné avec un constituant plus englobant. De manière plus surprenante, l'analyse lexicalisée de l'extraction proposée par Sag (1997), couplée avec une analyse des auxiliaires utilisant l'attraction d'arguments, autorise précisément de combiner un syntagme extrait avec un verbe dont l'un des dépendants est (ou contient) le site d'extraction.

24. Les constructions de ce type sont nombreuses. Par exemple, Bonami \& Godard (à paraître), Bonami et al. (1998) montrent que c'est le cas pour les phrases contenant un GN sujet inversé en français.

25. Pour des analyses de l'extraposition dans ces termes, cf. Dowty (1997) pour l'anglais, Reape (1994) et Kathol (1995) pour l'allemand.

26. Le choix de valeurs atomiques en GAP classiques était motivé entre autres par la nécessité de pouvoir comparer simplement les catégories pour le compactage, sans risquer de forcer des identifications de valeurs de traits non souhaitées. L'utilisation de structures de traits organisées hiérarchiquement règle le problème: dans le système présenté ci-après, seules les valeurs de TETE sont comparées pour le compactage, les autres traits n'étant pas affectés.

27. Cette hypothèse n'est pas innocente : en particulier, elle implique que dans une phrase, il n'y a pas de constituant GV intermédiaire entre $\mathrm{S}$ et V. Une telle position n'est pas problématique en GAP, dans la mesure où l'identité positionnelle distincte des sujets (ici, couleur 1) et des compléments (ici, le plus souvent, couleur 5) permet de les distinguer.

\section{RÉSUMÉS}

Cet article propose une comparaison de trois approches alternatives de l'ordre des mots dans les grammaires syntagmatiques, et en particulier en HPSG (Pollard \& Sag). Après une présentation des trois approches, je montre que certains phénomènes d'ordre récalcitrants en français et en allemand sont difficiles à traiter dans le formalisme classique des grammaires DI/PL (Gazdar, Klein, Pullum \& Sag), et demandent la reconnaissance de domaines d'ordre opaques à l'intérieur des constituants. Les grammaires d'arbres polychromes (Cori \& Marandin) et les grammaires de linéarisation directe (Kathol) sont mieux armées pour rendre compte de ce type de phénomène.

This paper compares three alternative approaches to word order in phrase-structure grammars, with a focus on HPSG (Pollard \& Sag). After presenting the three approaches, I show that some intriguing word order phenomena in French and German are difficult to account for in the mainstream framework of ID/LP grammars (Gazdar, Klein, Pullum \& Sag) and ask for a notion of opaque word order domain inside constituents. Polychrome tree grammars (Cori \& Marandin) and direct linearization grammars (Kathol) are better equipped to account for this type of phenomena. 
AUTEUR

OLIVIER BONAMI 